\title{
Visual binaries among high-mass stars
}

\section{An adaptive optics survey of OB stars in the NGC 6611 cluster}

\author{
G. Duchêne ${ }^{1,2, \star}$, T. Simon ${ }^{3, \star \star}$, J. Eislöffel ${ }^{4}$, and J. Bouvier ${ }^{1}$ \\ 1 Laboratoire d'Astrophysique de l'Observatoire de Grenoble, BP 53, 38041 Grenoble Cedex 9, France \\ 2 Division of Astronomy and Astrophysics, UCLA, Los Angeles, CA 90095-1562, USA \\ 3 Institute for Astronomy, University of Hawaii, 2680 Woodlawn Drive, Honolulu, HI 96822, USA \\ 4 Thüringer Landessternwarte Tautenburg, Sternwarte 5, 07778 Tautenburg, Germany
}

Received 27 June 2001 / Accepted 19 September 2001

\begin{abstract}
We have searched for visual binaries with projected separations in the range 200-3000 AU (0.' $\left.1-1^{\prime \prime} 5\right)$ among a sample of 96 stars in the massive young NGC 6611 cluster, 60 of them being subsequently identified as high probability cluster members of mainly OB spectral type. This is the first visual binary survey among such a large and homogeneous sample of high-mass stars. We find an uncorrected binary frequency of $18 \pm 6 \%$ over the surveyed separation range. Considering only binaries with mass ratios $q \geq 0.1$, we find that OB stars in NGC 6611 host more companions than solar-type field stars. We derive mass ratios for the detected binaries from their near-infrared flux ratios and conclude that about half of the detected binaries have $q \lesssim 0.2$, which does not contradict the assumption that companion masses are randomly drawn from the initial mass function. There is no evidence in our sample that wide-binary properties depend upon the mass of the primary star. The high frequency of massive binaries in a cluster as rich as NGC 6611 and the lack of a strong mass dependence of their properties are difficult to reconcile with the scenario whereby massive stars form as the result of mergers of smaller stars. The canonical protostellar accretion scenario together with cloud fragmentation, on the other hand, can naturally explain most of the observed binary properties, although the very high stellar density in the protocluster is likely to require significant modification to that picture as well.
\end{abstract}

Key words. stars: binaries: visual - stars: formation - stars: early-type - Galaxy: open clusters and associations: individual: NGC 6611

\section{Introduction}

Most detailed studies concerning star formation have focussed on low-mass stars $\left(M \lesssim 1 M_{\odot}\right)$, because they are more numerous and relatively nearby (Ghez et al. 1993; Leinert et al. 1993; Prosser et al. 1994; Simon et al. 1995; Petr et al. 1998). However, a growing effort is underway to incorporate high-mass stars $\left(M \gtrsim 10 M_{\odot}\right)$ in our understanding of this general process. The potentially major impact of such massive and short-lived stars includes their high photoionizing ultraviolet flux and mechanical feedback on the interstellar medium through strong mass-loss phenomena as well as dynamical interactions with neighbouring low-mass objects.

\footnotetext{
Send offprint requests to: G. Duchêne, e-mail: duchene@astro.ucla.edu

* Visiting astronomer, Canada-France-Hawaii Telescope Corporation operated by the National Research Council of Canada, the Centre National de la Recherche Scientifique de France and the University of Hawaii.

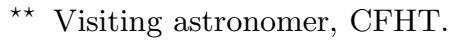

The canonical inside-out collapse model for the formation of low-mass objects (e.g., Shu et al. 1987) cannot account for the formation of massive stars due to the strong radiative flux from the central source that eventually overcomes the spherical accretion flow; the critical mass beyond which this occurs is $\sim 10 M_{\odot}$ (Beech \& Mitalas 1994). The formation of such massive objects thus requires another process in order to by-pass this hard limit. The apparent tendency of OB stars to prefer the cores of dense stellar clusters is not the result of rapid dynamic mass segregation (Hillenbrand \& Hartmann 1998; Bonnell \& Davies 1998) since it is observed in clusters which are younger than their relaxation times. Rather, a dense environment appears to be a needed condition for the formation of high-mass stars. A scenario that takes this factor into account has recently been presented by Bonnell et al. (1998), who suggested that these objects form through tidally-induced mergers of intermediate mass protostars in the early stages of the formation process. In this framework, where dynamical interactions play a major role, it 
is natural to study the properties of wide binaries in order to probe this mechanism, as pairs with separations larger than a few tens of $\mathrm{AU}$ are expected to be perturbed, if not disrupted, during close encounters with other protostars. As opposed to this violent process, the accretion paradigm could still be valid, provided it occurs in a geometrically thin equatorial disk, which would not be blown away by radiation pressure as easily as would a spherical envelope (Nakano 1989). Another alternative consists in spherical accretion of highly dust-depleted material (Wolfire \& Cassinelli 1987).

Because of their scarcity, OB stars are usually found far away from the Sun (several hundreds of parsecs or more) so that direct imaging surveys for visual binaries among the high-mass stars are strongly constrained by resolution issues and have only rarely been attempted so far. On the other hand, these stars offer many opportunities for spectroscopic surveys because of their extreme brightness. Garmany et al. (1980), Abt et al. (1990), and Morrell \& Levato (1991) have performed such surveys in the past and have all concluded that the proportion of spectroscopic binaries among massive stars is as large as that among solar-type stars, if not significantly higher. With the advent of fast readout detectors, speckle surveys of bright stars have also been conducted (McAlister et al. 1993; Mason et al. 1998) and revealed a non-negligible fraction of visual binaries among the OB stars, despite the limited dynamical range of this technique. Overall, taking into account various surveys probing different orbital period/separation ranges, it appears that OB stars are often, if not always, found in multiple systems. Other properties, such as orbital period and mass ratio distributions for the OB binaries, are not as easy to estimate, due to the limited sensitivity and the biases that are often inherent in inhomogeneous samples.

One way to ensure both the homogeneity of the sample and a uniform sensitivity to companions is to consider high-mass stars that are located in a stellar cluster. Spectroscopic surveys for binarity have already been conducted in several clusters (for a review, see Mermilliod \& García 2001) or young stellar associations (Morrell \& Levato 1991). Similar surveys for visual binaries among high-mass stars have only been performed in the Orion Trapezium cluster by Preibisch et al. (1999), though in a sample comprised of just 13 OB stars. Other massive spectroscopic binaries in OB associations are known, but were not discovered in the course of large-scale surveys (e.g., Gieseking 1982). To derive accurate statistical properties for massive visual binaries, one must consider much richer clusters that host several tens of OB stars.

NGC 6611 is the young, massive stellar cluster that is responsible for the photoionization of the Eagle Nebula, famous for its Elephant Trunks (Duncan 1920). The first in-depth study of the cluster was performed by Walker (1961) in his pioneering work on star-forming regions. He showed that NGC 6611 is an extremely young cluster containing a pre-main sequence population of intermediatemass objects, of spectral type later than B5, together

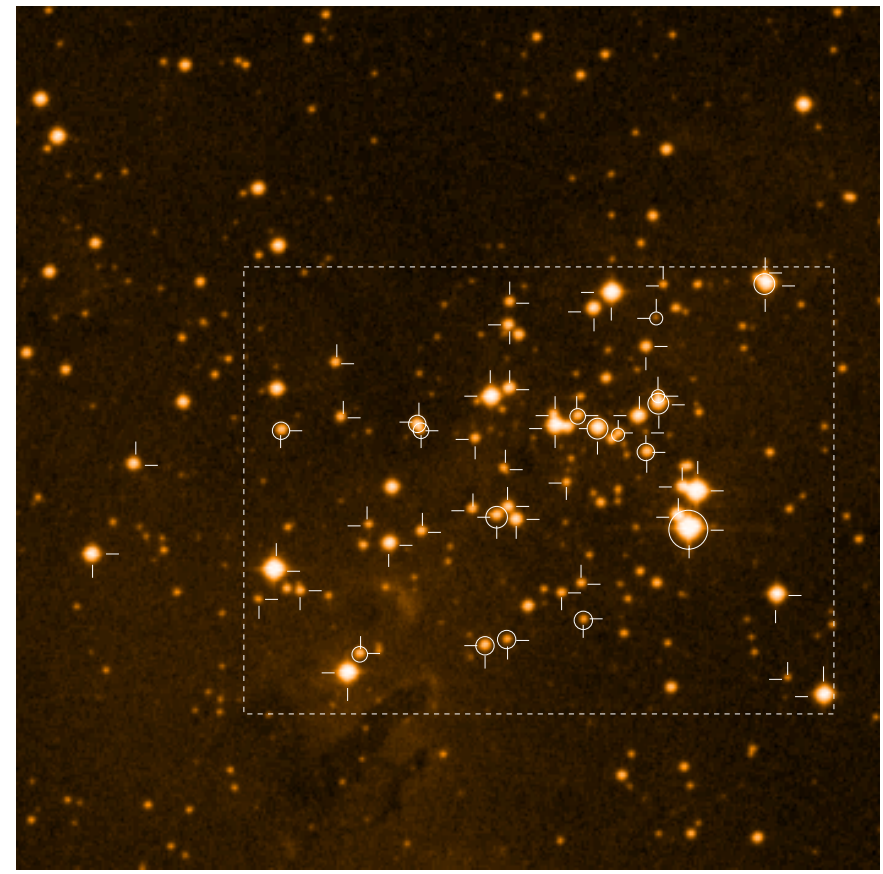

Fig. 1. Digital Sky Survey $10^{\prime} \times 10^{\prime}$ image of the central part of the NGC 6611 cluster. Stars marked with ticks are cluster members observed in this survey (including the faint underexposed members), while circles identify all the newly discovered pairs with separations smaller than 1". 5 , both physical and likely background companions. The dashed box, which encloses the core of the cluster, contains about $75 \%$ of the cluster members of spectral type B0 or earlier. Another dozen members, including three binary systems, were observed outside the core of the cluster; most of these lie outside of this image.

with several tens of higher mass main sequence objects. Deeper studies of the cluster were subsequently performed by Thé et al. (1990), Hillenbrand et al. (1993), de Winter et al. (1997) and Belikov et al. (1999, 2000). There is a general consensus for a variable visual extinction, ranging from 1 to $5 \mathrm{mag}$, and an anomalous extinction law towards the cluster (e.g., de Winter et al. 1997), while other properties, such as the age and distance of the cluster, remain somewhat uncertain. Estimates for the distance to the cluster depend on the extinction law assumed and have been given as $2.0 \mathrm{kpc}$ (Hillenbrand et al. 1993), $2.1 \mathrm{kpc}$ (McBreen et al. 1982; Belikov et al. 1999), $2.3 \mathrm{kpc}$ (Meaburn \& White 1982, average value from anterior studies) and $2.6 \mathrm{kpc}$ (Thé et al. 1990). The age of the cluster is also debated, but its extreme youth (at most a few Myr) is evidenced by the presence of some very massive stars: the star of earliest spectral type, W 205, is an O5 main sequence object. The median age of the cluster is of the order 2-3 Myr (Hillenbrand et al. 1993; Belikov et al. 2000), but the age spread within the cluster is perhaps as large as $6 \mathrm{Myr}$ (de Winter et al. 1997; Belikov et al. 2000). Throughout this paper, we adopt the results of Hillenbrand et al. (1993), i.e. an age of $2 \mathrm{Myr}$ and a distance of $2 \mathrm{kpc}$. 
Table 1. Cluster members observed (Main Sample) together with our $K$-band photometry. A ${ }^{\S}$ symbol denotes the stars that are located outside the central area of the cluster; membership probabilities (Cols. 3 and 9) are from Belikov et al. (1999) and $V$ magnitudes from Hillenbrand et al. (1993); 39 of these stars are "very likely" $(3 \sigma)$ members following Belikov et al. (1999). References for the spectral classification: 1 - Hillenbrand et al. (1993); 2 - de Winter et al. (1997); 3 - Thé et al. (1990); 4 - Bosch et al. (1999).

\begin{tabular}{|c|c|c|c|c|c|c|c|c|c|c|c|}
\hline $\begin{array}{l}\text { Walker } \\
\text { number }\end{array}$ & $K$ & $\begin{array}{c}\text { memb. } \\
{[\%]}\end{array}$ & $V$ & S.T. & ref. & $\begin{array}{l}\text { Walker } \\
\text { number }\end{array}$ & $K$ & $\begin{array}{c}\text { memb. } \\
{[\%]}\end{array}$ & $V$ & S.T. & ref. \\
\hline $25^{\S}$ & 10.56 & 53 & 12.93 & B0.5 & 1 & $290^{\S}$ & 10.82 & 52 & 12.14 & B2 & 2 \\
\hline $114^{\S}$ & 13.70 & 32 & 16.11 & - & - & 296 & 10.16 & 95 & 11.78 & B2 & 3 \\
\hline $125^{\S}$ & 8.53 & 79 & 10.01 & B1 & 1 & 299 & 12.75 & 55 & 14.46 & B6 & 2 \\
\hline 150 & 8.28 & 86 & 9.85 & B0.5 & 1 & 300 & 10.94 & 16 & 12.69 & B1.5 & 1 \\
\hline 162 & 12.99 & 28 & 15.27 & - & - & 305 & 9.77 & 84 & 13.51 & B1 & 4 \\
\hline 166 & 8.60 & 95 & 10.36 & O8.5 & 1 & 306 & 10.46 & 96 & 12.77 & B1.5 & 1 \\
\hline 175 & 7.07 & 95 & 10.09 & O5.5 & 1 & 307 & 11.08 & 76 & 14.18 & B1.5 & 1 \\
\hline 177 & 11.57 & 46 & 15.33 & - & - & 311 & 11.29 & 51 & 13.10 & B2.5 & 1 \\
\hline $188^{\S}$ & 8.82 & 68 & 13.13 & B0 & 1 & 313 & 11.23 & 90 & 12.92 & B4 & 2 \\
\hline 197 & 7.31 & 46 & 8.73 & O7 & 1 & 314 & 7.93 & 98 & 9.85 & B0 & 1 \\
\hline 205 & 6.80 & 41 & 8.18 & O5 & 1 & 322 & 11.41 & 26 & 13.68 & B8 & 1 \\
\hline 207 & 10.19 & 89 & 12.07 & B1 & 1 & 323 & 11.59 & 58 & 13.48 & B5 & 1 \\
\hline 210 & 9.95 & 92 & 11.68 & B1 & 1 & 336 & 11.51 & 88 & 13.29 & B3 & 2 \\
\hline 221 & 11.01 & 92 & 14.55 & B8 & 1 & 339 & 10.85 & 95 & 13.74 & B3 & 2 \\
\hline 223 & 9.48 & 89 & 11.20 & B1 & 1 & 343 & 8.91 & 83 & 11.72 & B1 & 1 \\
\hline 224 & 10.96 & 96 & 14.74 & - & - & 351 & 9.68 & 91 & 11.26 & B1 & 1 \\
\hline 227 & 10.55 & 89 & 12.85 & B1.5 & 1 & 360 & 10.76 & 74 & 14.48 & - & - \\
\hline 231 & 10.04 & 78 & 12.71 & B1 & 1 & 367 & 8.75 & 76 & 9.39 & O9.5 & 1 \\
\hline 235 & 7.93 & 96 & 10.98 & B1 & 3 & 371 & 11.19 & 72 & 13.44 & B0.5 & 1 \\
\hline 243 & 11.61 & 93 & 13.80 & B8 & 1 & 374 & 10.30 & 19 & 13.41 & $\mathrm{~F} 2$ & 2 \\
\hline 246 & 6.66 & 88 & 9.46 & O7 & 1 & 388 & 11.81 & 90 & 13.70 & B6 & 2 \\
\hline 254 & 9.64 & 99 & 10.80 & B1 & 1 & 400 & 10.44 & 82 & 12.87 & B8 & 2 \\
\hline 259 & 9.20 & 87 & 11.61 & B0.5 & 1 & 401 & 7.59 & 46 & 8.90 & O8.5 & 1 \\
\hline 260 & 12.38 & 44 & 14.38 & - & - & $412^{\S}$ & 7.26 & 34 & 8.18 & O9.5 & 1 \\
\hline 262 & 11.67 & 68 & 13.96 & B7 & 2 & $468^{\S}$ & 8.57 & 47 & 9.40 & B1 & 1 \\
\hline 267 & 11.47 & 87 & 13.13 & B2 & 2 & $469^{\S}$ & 9.32 & 70 & 10.69 & B0.5 & 1 \\
\hline 273 & 11.68 & 58 & 14.21 & $\mathrm{~A} 0$ & 2 & $483^{\S}$ & 9.51 & 72 & 10.99 & B4.5 & 2 \\
\hline 276 & 11.39 & 69 & 13.74 & B5 & 2 & $489^{\S}$ & 10.11 & 71 & 11.57 & B7 & 2 \\
\hline 280 & 8.72 & 98 & 10.12 & O9.5 & 1 & $503^{\S}$ & 7.86 & 40 & 9.75 & B0-B0.5 & 3 \\
\hline 281 & 11.86 & 96 & 13.80 & A & 1 & $536^{\S}$ & 10.21 & 39 & 11.46 & $\mathrm{~B} 1$ & 1 \\
\hline
\end{tabular}

The first systematic, though limited, search for binaries in NGC 6611 was performed by Bosch et al. (1999), who conducted a spectroscopic survey of the 10 earliest type stars in the cluster. In this paper, we present the first high-angular resolution imaging binary survey of the OB population of NGC 6611, which is an extension of our low-mass binary surveys in young clusters (Bouvier et al. 1997; Duchêne et al. 1999; Bouvier et al. 2001). We aim at (i) estimating some of the binary properties with which we can then confront the collisional formation model of high-mass stars, and (ii) comparing directly these properties to those that have been previously estimated for lower mass binaries. The sample and observations are described in Sect. 2; Sect. 3 describes our results and compares them to previous OB multiplicity surveys. Implications for the scenarios of massive star formation are then considered in Sect. 4. Finally, Sect. 5 summarizes the main results of our study.

\section{Sample selection and observations}

\subsection{Sample definition}

Assessing membership for stars in NGC 6611 is an especially crucial issue in view of the location of the cluster near the Galactic plane $(l \sim 17 \mathrm{deg}, b \sim 0$.8), its large distance, and its moderate intracluster extinction. Numerous foreground low-mass dwarfs as well as bright background giants are likely to be projected on the cluster and thus confused with true cluster members. Furthermore, the intrinsic proper motion of the cluster is very small ( $\sim 2.5 \mathrm{mas} / \mathrm{yr}$, Belikov et al. 1999) and not well differentiated from that of the field stars. Combining proper motion measurements with the spatial location of the stars, Belikov et al. (1999) estimated individual membership probabilities for all stars brighter than $V=16.8$. Most of the stars with spectral type earlier than B5 were thus confirmed as cluster members, providing a robust 
Table 2. List of additional stars observed in our programme. The notes in Cols. 7 and 14 indicate the reason why the star is excluded from our sample: "n" means that the star is not a likely cluster member, while "f" indicates that the exposure time used during the observation was insufficient to ensure a good detection limit. KS numbers are from Kharchenko \& Schilbach (1995); other columns have the same meaning as in Table 1.

\begin{tabular}{|c|c|c|c|c|c|c|c|c|c|c|c|c|c|}
\hline object & $K$ & $\begin{array}{c}\text { memb. } \\
{[\%]}\end{array}$ & $V$ & S.T. & ref. & note & object & $K$ & $\begin{array}{c}\text { memb. } \\
{[\%]}\end{array}$ & $V$ & S.T. & ref. & note \\
\hline $\mathrm{W} 136^{\S}$ & 13.23 & 0 & 15.94 & - & - & $\mathrm{n}$ & W 285 & 12.87 & 4 & 15.16 & - & - & $\mathrm{n}$ \\
\hline W 181 & 11.21 & 7 & 14.32 & B1.5 & 1 & $\mathrm{n}$ & W 297 & 10.62 & 10 & 12.88 & B1.5 & 1 & $\mathrm{n}$ \\
\hline W 182 & 11.74 & 8 & 16.29 & - & - & $\mathrm{n}, \mathrm{f}$ & W 301 & 10.42 & 92 & 15.49 & B2 & 1 & $\mathrm{f}$ \\
\hline W 198 & 11.56 & 0 & 13.21 & - & - & $\mathrm{n}$ & W 309 & 11.16 & 0 & 15.49 & - & - & $\mathrm{n}$ \\
\hline W 202 & 11.19 & 0 & 14.40 & B3 & 2 & $\mathrm{n}$ & W 321 & 9.70 & 0 & 15.33 & - & - & $\mathrm{n}$ \\
\hline W 209 & 12.79 & 0 & 15.81 & - & - & $\mathrm{n}, \mathrm{f}$ & W 335 & 12.71 & 0 & 16.58 & - & - & $\mathrm{n}, \mathrm{f}$ \\
\hline W 213 & 9.48 & 0 & 14.18 & $\mathrm{~A} 7-\mathrm{F} 9$ & 2 & $\mathrm{n}$ & W 353 & 14.45 & 0 & 16.55 & - & - & $\mathrm{n}, \mathrm{f}$ \\
\hline W 226 & 13.22 & 14 & 15.88 & - & - & $\mathrm{f}$ & W 355 & 13.49 & 0 & 15.71 & - & - & $\mathrm{n}, \mathrm{f}$ \\
\hline W 229 & 13.38 & 30 & 16.20 & - & - & $\mathrm{f}$ & W 362 & 13.41 & 2 & 15.89 & - & - & $\mathrm{n}$ \\
\hline W 237 & 12.41 & 2 & 15.17 & - & - & $\mathrm{n}$ & W 364 & 11.36 & 74 & 13.44 & - & - & $\mathrm{f}$ \\
\hline W 240 & 12.54 & 6 & 14.56 & B8 & 2 & $\mathrm{n}$ & W 366 & 11.00 & 0 & 14.27 & - & - & $\mathrm{n}$ \\
\hline W 245 & 9.79 & 1 & 13.61 & B6 & 2 & $\mathrm{n}$ & W 396 & 10.39 & 3 & 14.03 & F9-G2 & 2 & $\mathrm{n}, \mathrm{f}$ \\
\hline W 252 & 11.44 & 0 & 13.90 & - & - & $\mathrm{n}$ & $\mathrm{W} 455^{\S}$ & 10.53 & 7 & 12.11 & B7 & 2 & $\mathrm{n}$ \\
\hline W 257 & 12.51 & 0 & 15.59 & - & - & $\mathrm{n}$ & $\mathrm{W} 460^{\S}$ & 12.48 & 0 & 15.36 & - & - & $\mathrm{n}, \mathrm{f}$ \\
\hline W 258 & 12.65 & 0 & 15.43 & - & - & $\mathrm{n}$ & $\mathrm{W} 487^{\S}$ & 12.40 & 12 & 15.00 & - & - & $\mathrm{n}$ \\
\hline W 266 & 9.61 & 0 & 14.35 & F8 & 2 & $\mathrm{n}$ & KS 29617 & 11.47 & 0 & 16.30 & - & - & $\mathrm{n}, \mathrm{f}$ \\
\hline W 270 & 12.83 & 0 & 15.55 & - & - & $\mathrm{n}, \mathrm{f}$ & KS 29710 & 11.60 & 37 & 11.48 & - & - & $\mathrm{f}$ \\
\hline W 275 & 10.64 & 0 & 12.12 & B1.5 & 1 & $\mathrm{n}$ & KS $29797^{\S}$ & 14.46 & 4 & 16.03 & - & - & $\mathrm{n}$ \\
\hline
\end{tabular}

sample for a statistical study of binarity in this cluster. Belikov et al. (2000) further used optical photometry data to reassess cluster membership. Statistically, about $75-$ $80 \%$ of their sample was confirmed as belonging to the cluster. On the other hand, the low-mass T Tauri population of NGC 6611 is totally unknown due to the distance to the cluster and the extreme crowding of the field for fainter stars.

Our sample, drawn from the lists of Walker (1961) and of Kharchenko \& Schilbach (1995), contains 59 OB stars as well as about twenty intermediate-type objects. Most of these objects (66 out of 82 targets) are located in the central area of the cluster, which contains most of the O-type stars of the cluster (this area is defined in Fig. 1). An additional fourteen objects from the lists mentioned above were detected in our images, but were not exposed long enough to ensure a satisfactory companion detectability, and so are not considered further.

We use the membership probabilities derived by Belikov et al. (1999), which were published after our observations were completed, to identify cluster members. These authors set two probability thresholds at $14 \%$ and $61 \%$, which correspond respectively to "likely" $(2 \sigma)$ and "very likely" $(3 \sigma)$ cluster members. In the following, our Main Sample consists of all sources having membership probabilities larger than $14 \%$ and that were bright enough to ensure the detection of a 6 mag flux ratio companion at $1^{\prime \prime}$; it contains $51 \mathrm{OB}$ stars and a few later-type objects. The 60 members that form our Main Sample are listed in Table 1, while the remaining 36 targets are presented in Table 2, including "faint" stars. The observed members located in the core of the cluster are indicated in Fig. 1, as are the newly discovered binary systems. Only two members located in the core and brighter than $V=14.5$ were not observed in this survey (W 349 and W 402); both are late G-type (super)giants according to de Winter et al. (1997).

\subsection{Observations and data reduction}

We used the adaptive optics system, PUEO (Rigaut et al. 1998), at the Canada-France-Hawaii Telescope with the $1024 \times 1024$ near-infrared detector KIR (Doyon et al. 1998) during four nights in June and July 1998. The pixel size is about 0 .'035, yielding a total field of view of $36^{\prime \prime}$. Each target employed for wavefront sensing was observed in a four-step dithered pattern, which increased the field of view to a final image size of about $50^{\prime \prime}$ and generally resulted in the detection of several cluster members per field. Total integration varied from $3 \mathrm{~s}$ to 15 min depending on the source brightness and field crowding. Data reduction included all the usual steps for near-infrared images (sky subtraction, flat-fielding and shift-and-add), and was performed with standard IRAF ${ }^{1}$ routines.

To deal with demanding contrast issues, we conducted the survey in the $K$-band, since high-mass stars fade much more rapidly at longer wavelengths than do their putative low mass secondaries. Our images revealed a large number

\footnotetext{
${ }^{1}$ IRAF is distributed by the National Optical Astronomy Observatories, which is operated by the Association of Universities for Research in Astronomy, Inc., under contract to the National Science Foundation.
} 
of stars, up to several hundred in each field, due to the low galactic latitude of the cluster. The tight pairs detected in the raw images at the telescope were immediately observed in the $J$ and $H$ bands. Some of the binaries identified in this survey are shown in Fig. 2. Figure 3 presents a plot of the $K$-band differential photometry for the companions that were detected at $K$, as well as our detection limit for that wavelength. The latter is an azimuthally averaged value, which has been estimated by adding artificial faint companions to the images of single stars. Figure 3 also shows the impact of a change in image quality on the detectability of companion stars. Such a change mostly affects the inner 0.5 , where the diffraction-limited part of the stellar point spread function (PSF) dominates the flux. Our detection limit has further been empirically confirmed by combining all companions detected with the same instrumentation in the course of our survey of young clusters (Bouvier \& Duchêne 2001).

Some of the companions detected in the $K$-band were undetected in the $J$-band, and sometimes even in the $H$-band (e.g., W 275 and W 297). This is the result of two complementary effects: (i) the intrinsic brightness difference between the two stars increases strongly towards shorter wavelengths, and (ii) the adaptive optics correction degrades when observing at the shorter wavelengths, especially in the $J$-band, thereby increasing the flux in the halo of the primary. We were able to estimate minimum flux ratios in those cases by subtracting the PSF of the primary and then extracting a flux limit by measuring the noise level at the known location of the secondary.

Aperture photometry was performed through $2^{\prime \prime}$ radius apertures and then absolute calibration in the CIT system was obtained by comparison to the Hillenbrand et al. (1993) photometry of the cluster area. Based on the comparison of 40 to 80 independent measurements in each filter, the standard deviation between the two datasets is $\lesssim 0.1 \mathrm{mag}$, including possible long-term variability for some stars. Binary flux ratios, separations and position angles were usually obtained through PSF fitting and/or aperture photometry for wide enough systems. Flux ratio uncertainties are estimated by comparing various images of the same system; it appears to be 0.03 mag in both cases. We employed a Richardson-Lucy deconvolution algorithm for the tighest pairs and subtracted the PSF of the primary star for those companions that were just barely above our detection limit. The two methods in principle require perfect knowledge of the PSF; both yield uncertainties of the order of 0.1 mag. Typical uncertainties in our astrometric measurements are about 0.005 for the separation and 0.2 for the position angle on the sky, half coming from the absolute calibration, which was derived from observations of several well-known binaries from the Index Catalogue of Double Stars (van Dessel \& Sinachopoulos 1993). In the few cases where the secondary was barely above our detection limit, these uncertainties can reach $0^{\prime \prime} 01$ and up to $4^{\circ}$ for the tightest pairs. Five binaries are affected by these larger uncertainties, namely, W 205, W 213, W 223, W 224 and W 260.

\section{Results}

Because most of our images contain large numbers of sources, we cannot a priori consider a random pair of stars as being a physical binary system; we first have to set a criterion to exclude very wide pairs and then check that the remaining systems are indeed physically linked. We first adopt a 1".5 upper limit for considering pairs, both because this corresponds to a fairly large physical separation (3000 AU) at the distance of NGC 6611 and because the average distance to the nearest neighbour down to $K \approx 18.5$ is about $1^{\prime \prime} .4$ in our most crowded field. Photometric and astrometric measurements for all detected pairs are summarized in Table 3 where seven candidate background companions, which are identified in this section, are marked.

Before we discuss statistical properties of the highmass binary population of NGC 6611, it is important to determine which of these pairs indeed form bound systems (Sect. 3.1). In this section, we also estimate the binary mass ratios (Sect. 3.2) and derive a lower limit for the total binary frequency of OB stars in NGC 6611 and compare this value to previous binary surveys (Sect. 3.3). Finally, we summarize other important trends (Sect. 3.4).

\subsection{Bound companions}

We must consider two aspects to confirm the physical status of the identified pairs. First, the two stars must be cluster members located at the same depth in the cloud, as opposed to two unrelated members that merely appear close together on the sky due to projection effects. When this is secured (Sect. 3.1.1), we have to check that such wide pairs form bound systems. The latter point can be argued from studies of lower-mass stars: $3000 \mathrm{AU}$ is a safe upper limit for separations of solar-type binaries (e.g., Duquennoy \& Mayor 1991). Furthermore, Abt (1988) estimated a maximum separation threshold of about 20000 AU for B5 primaries and it is likely that more massive primaries sustain even wider systems. We thus infer that any cluster star physically located within $3000 \mathrm{AU}$ of another high-mass cluster star can be safely interpreted as a bound companion. However, two cluster members can be projected next to each other on the sky while being at different depths in the cluster. A quantitative estimate of such cases is presented in Sect. 3.1.2, simultaneously considering nonmembers as possible false companions.

\subsubsection{Near-infrared properties of detected companions}

First, we infer membership for the putative secondaries by plotting all components of the detected binaries in a near-infrared $(K, H-K)$ colour-magnitude diagramme. As can be seen in Fig. 4 , the primaries of the known members of the cluster form a vast cloud of points. About half the secondaries also lie in the same locus, suggesting that they really are cloud members. However, a few companions are isolated from other members in this diagramme. 

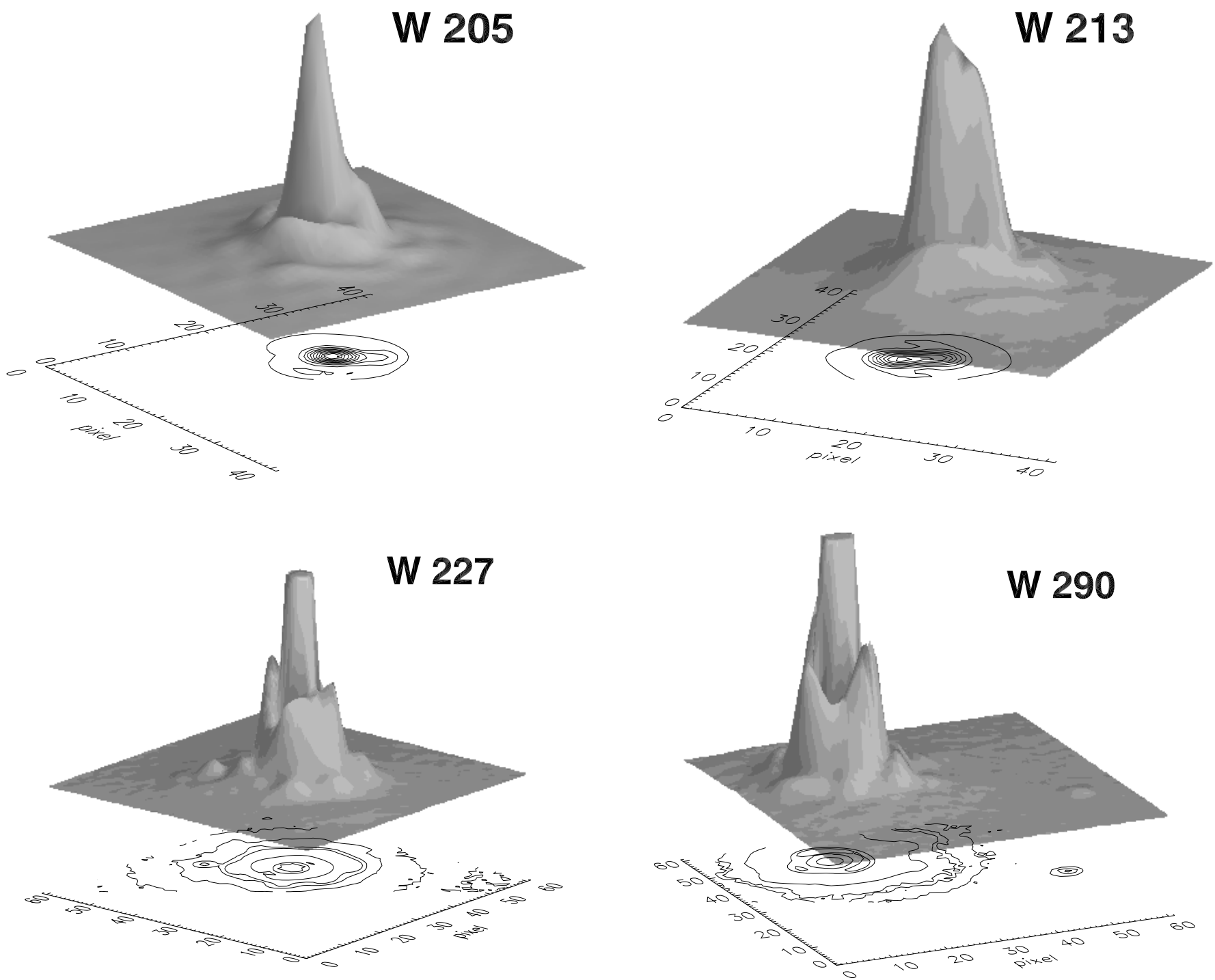

Fig. 2. Surface and contour plots of some of the binaries detected in this survey, illustrating the resolving power of our observing technique. The upper two images are 1". 4 on a side and represents the tightest pairs that we found, while the lower ones are 2 '. 1 wide and illustrate large flux ratios in wider systems. All images were obtained in the $K$-band. Note the excellent adaptive optics correction in the image of W 290, in which the first Airy ring is almost azimuthaly symetric as well as the clear detection of the companion although the binary flux ratio is $\Delta K \approx 5.9 \mathrm{mag}$. On the other hand, the companion to W 227 (to the left of the primary in this image) appears barely above the noise in the imperfect adaptive optics halo.

They could be either highly extincted low-mass members of the cluster or else heavily reddened objects in the background that are seen through the molecular cloud. One way to distinguish between these two possibilities is to compare the apparent extinction needed to bring the primaries and their secondaries back onto the same theoretical isochrone, assuming both stars have the same age. Since both stars are equally embedded in the molecular cloud, one expects both components of a system to show a similar extinction, unless one of them suffers a significant circumstellar extinction. Consequently, secondaries which appear much more extincted than their primaries are serious background giant candidates. Five of the seven such candidates identified in Table 3 are excluded by virtue of this criterion; in each of these cases, the extinction $A_{V}$ towards the secondary appears to be at least 5 mag larger than that along the line of sight to the primary.

In addition, when a "companion" is detected in the $J$-band, one can also use the $J-H$ colour index to confirm its status: Hillenbrand et al. (1993) suggested that objects with $J-H>1.6$ likely lie beyond the cluster. Among the five sources mentioned above, two display $J-H>1.6$ (W 243 and W 339), while the other three are not detected in the $J$-band, but have suspicious $H-K$ colour indexes $(H-K>0.85)$. Since none of the other companions shows such extremely red colours, we conclude that these five 


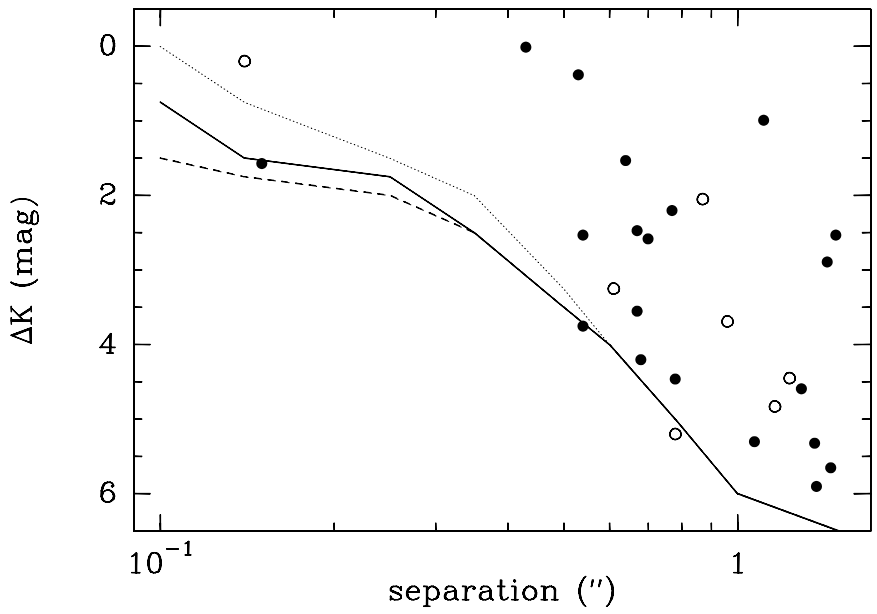

Fig. 3. Detected companions around our targets and overall detection limit of our survey. Plotted is the $K$-band flux ratio as a function of the binary separation. Filled circles denote close companions to cluster members (including around the "faint" members W 226 and W 364) found in this survey, while open circles denote non-members. The solid line is our typical detection limit while dashed and dotted curves correspond respectively to the best and worst image quality achieved in our survey.

objects are all very likely to be background giants ${ }^{2}$. Beyond our Main Sample, the companion to W 226 is also a likely background object while W 364 seems to be a physical system. We also emphasize that both of these criteria help identifying background stars but do not provide any evidence for/against the presence of projected foreground objects.

\subsubsection{Statistical contamination by field stars}

We consider here the possibility that some of the close pairs simply result from random pairing of field stars. To do so, we first compute the average spatial density of objects that are at least as bright as the companion in the image. We then estimate the probability of finding one such object at a distance from the primary at most equal to that of the companion.

Equivalently, we estimate here $P_{\text {bound }}$, the complementary quantity to this probability, that no random star brighter than the secondary $\left(K_{\mathrm{p}} \leq K \leq K_{\mathrm{s}}\right)$ is detected within the binary separation $r_{\mathrm{b}}$ from the primary, as being $P(N=0, \mu=\bar{N})$ derived from Poisson statistics. Our detection limit for companions is explicitely taken into

${ }^{2}$ A. Ghez and C. McCabe subsequently obtained a lowresolution long slit $K$-band spectrum of W 243 with NIRPSEC behind the adaptive optics system at W. M. Keck Observatory, with the slit oriented along the binary. The spectrum of the secondary revealed no significant feature, suggesting a spectral type $\mathrm{F}$ or earlier, inconsistent with its photometric mass estimates (see Table 4). This confirms the classification of this companion as a background star.

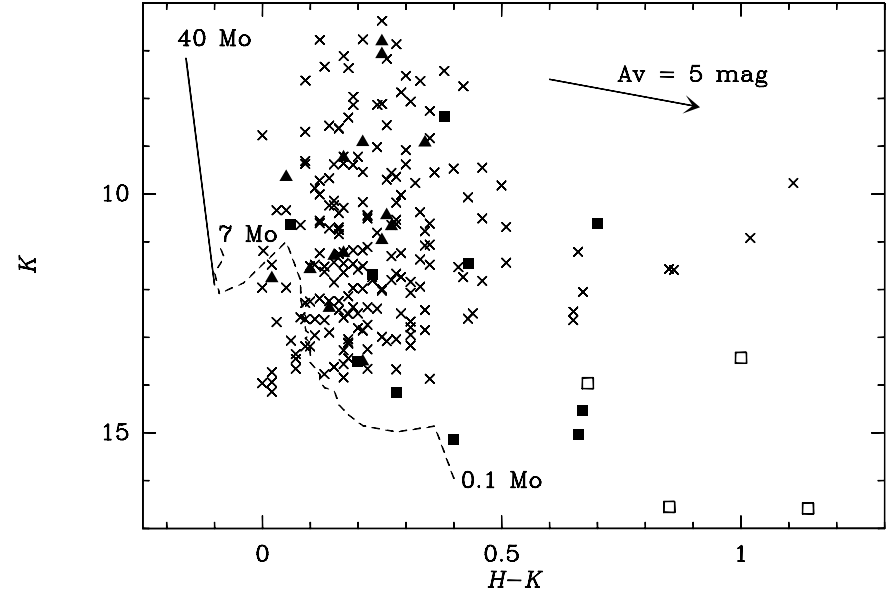

Fig. 4. Near-infrared colour-magnitude diagramme for NGC 6611. All likely $(2 \sigma)$ members from Belikov et al. (1999) are presented as crosses, using photometry from Hillenbrand et al. (1993). Filled triangles and squares represent member primaries and secondaries of binaries that were detected in our Main Sample. Suspected background secondaries are shown as open squares; the background companion to W 343, with its $H-K \approx 1.8 \mathrm{mag}$ colour index, is not plotted here. The solid line is a linear fit to the synthetic colours of Schaller et al. (1992) for high mass stars $\left(M>4 M_{\odot}\right)$, while the dashed curve shows the pre-main sequence evolutionary models of Siess et al. (2000) for stars with masses $0.1 M_{\odot}<M<7 M_{\odot}$; both models correspond to a 2 Myr cluster age. The local maximum at $H-K \approx 0.05$ corresponds to a mass of $3 M_{\odot}$, above which stars have already reached the ZAMS. Deuterium burning in very low-mass stars is responsible for the plateau at $0.2<H-K<0.4$. A typical extinction vector following the canonical law of Rieke \& Lebofsky (1985) is also shown.

account as we use the following expression for $\bar{N}$ :

$\bar{N}=\sum_{K=K_{\mathrm{p}}}^{K_{\mathrm{s}}} n_{K} W_{K}$

where $n_{K}$ and $W_{K}$ are respectively the cluster surface density in the considered field and the detectability area of stars at a given magnitude $K$, computed as follows:

$W_{K}=\int_{0 \prime \prime 1}^{r_{\mathrm{b}}} V_{K}(r) \times 2 \pi r \mathrm{~d} r$

We are assuming that the visibility $V_{K}(r)$ is simply

$V_{K}(r)=H\left(r-r_{K}\right)=\left\{\begin{array}{l}0 \text { if } r<r_{K} \\ 1 \text { if } r \geq r_{K}\end{array}\right.$

where $r_{K}$ corresponds to the minimum binary separation at which, according to Fig. 3, a secondary of infrared magnitude $K$ is detectable in our images. Therefore, it follows that $W_{K}=\pi\left(r_{\mathrm{b}}^{2}-r_{K}^{2}\right)$. The derived values for $P_{\mathrm{bound}}$ are listed in the last column of Table 3.

Although most systems appear likely bound $\left(P_{\text {bound }}>\right.$ $95.5 \%$, corresponding to a $2 \sigma$ confidence level, for 15 out of 18 binary members), the probability that all pairs are physically linked is only $41 \%$, thus urging for a deeper analysis. For six pairs, the probabilities of being physical are higher than $99.7 \%$ ( $3 \sigma$ confidence level). These 
Table 3. Observed properties of all detected binaries in NGC 6611 with separations smaller than $1^{\prime \prime} 5$. The last two columns give the number of stars brighter than the secondary in the same field and our empirical estimate of the probability that a system is physically bound. A symbol indicates that the apparent companion is a likely background giant given its apparent extinction (see Sect. 3.1) while a ${ }^{\S}$ flags those companions with suspiciously low mass (Sect. 3.2); all other systems have probabilities of being real which are above $95.5 \%$ ( $2 \sigma$ confidence level). The field containing the two systems indicated by $\mathrm{a}^{\dagger}$ symbol were observed under non-photometric conditions and we used the Hillenbrand et al. (1993) unresolved photometry together with our observed flux ratios for the analysis.

\begin{tabular}{|c|c|c|c|c|c|c|c|c|c|c|}
\hline $\begin{array}{l}\text { Walker } \\
\text { number }\end{array}$ & $\begin{array}{l}\text { sep. } \\
{\left[{ }^{\prime \prime}\right]}\end{array}$ & $\begin{array}{l}\mathrm{PA} \\
{\left[{ }^{\circ}\right]}\end{array}$ & $\overline{K_{\mathrm{A}}}$ & $H_{\mathrm{A}}$ & $J_{\mathrm{A}}$ & $\Delta K$ & $\Delta H$ & $\Delta J$ & $\begin{array}{c}N\left(K \leq K_{\mathrm{B}}\right) \\
\text { in field }\end{array}$ & $P_{\text {bound }}$ \\
\hline \multicolumn{11}{|c|}{ Main Sample binaries } \\
\hline 25 & 1.432 & 288.2 & 10.56 & - & - & 2.89 & - & - & 7 & 0.985 \\
\hline 175 & 0.672 & 231.1 & 7.07 & 7.32 & - & 3.55 & $4.0 \pm 0.1$ & - & 1 & 0.999 \\
\hline 188 & 0.543 & 227.7 & 8.92 & 9.26 & 9.66 & 2.53 & 2.62 & 2.80 & 1 & 0.999 \\
\hline 205 & 0.148 & 320.0 & 6.80 & 7.05 & - & $1.57 \pm 0.1$ & $1.7 \pm 0.1$ & - & 1 & 0.999 \\
\hline $223^{\dagger}$ & 1.081 & 295.0 & 9.49 & 10.07 & 9.95 & $5.3 \pm 0.1$ & $5.8 \pm 0.2$ & $>6.1$ & 48 & 0.955 \\
\hline $224^{\dagger}$ & 0.779 & 321.9 & 10.99 & 11.73 & 11.97 & 4.46 & 4.59 & $>4.5$ & 79 & 0.963 \\
\hline 227 & 0.538 & 29.8 & 10.58 & - & - & 3.75 & - & - & 46 & 0.991 \\
\hline 243 & 0.770 & 224.5 & 11.76 & 11.78 & 12.12 & 2.20 & 2.86 & $4.5 \pm 0.1$ & 28 & 0.975 \\
\hline 254 & 1.107 & 184.9 & 9.64 & 9.69 & 9.82 & 0.99 & 1.00 & 1.05 & 3 & 0.997 \\
\hline 260 & 0.676 & 152.6 & 12.38 & 12.52 & 12.78 & $4.2 \pm 0.1$ & $5.2 \pm 0.1$ & $>6.0$ & 107 & 0.962 \\
\hline 267 & 0.701 & 136.3 & 11.57 & 11.67 & 11.80 & 2.58 & 2.76 & $3.9 \pm 0.1$ & 48 & 0.974 \\
\hline $290^{\S}$ & 1.373 & 235.3 & 10.82 & - & - & $5.9 \pm 0.1$ & - & - & 134 & 0.793 \\
\hline 299 & 0.433 & 94.7 & 13.50 & 13.71 & 14.09 & 0.01 & 0.00 & -0.03 & 13 & 0.998 \\
\hline 311 & 0.530 & 254.3 & 11.29 & 11.44 & 11.80 & 0.38 & 0.46 & 0.47 & 5 & 0.998 \\
\hline $313^{\text {ब }}$ & 1.364 & 297.0 & 11.23 & 11.40 & 11.61 & 5.32 & $6.0 \pm 0.2$ & $>6.5$ & 123 & 0.783 \\
\hline 339 & 0.670 & 306.8 & 10.96 & 11.21 & 11.74 & 2.47 & 3.22 & $>4.5$ & 15 & 0.992 \\
\hline $343^{\pi}$ & 1.449 & 89.6 & 8.91 & 9.12 & 9.52 & 5.65 & $7.3 \pm 0.3$ & $>6.5$ & 41 & 0.921 \\
\hline $400^{\S}$ & 1.288 & 320.3 & 10.44 & 10.70 & 11.04 & 4.59 & 4.99 & $5.9 \pm 0.2$ & 51 & 0.900 \\
\hline \multicolumn{11}{|c|}{ Binaries among "faint members" } \\
\hline $226^{\top}$ & 0.643 & 346.1 & 13.46 & 13.40 & 13.87 & 1.53 & 2.18 & $3.9 \pm 0.2$ & 52 & 0.986 \\
\hline 364 & 1.484 & 267.8 & 11.36 & 11.58 & 11.75 & 2.53 & 2.87 & 3.62 & 16 & 0.960 \\
\hline \multicolumn{11}{|c|}{ Non-member primaries } \\
\hline 213 & 0.139 & 298.7 & 10.14 & 10.49 & 11.19 & $0.2 \pm 0.1$ & $0.2 \pm 0.1$ & $0.2 \pm 0.1$ & 1 & 0.999 \\
\hline 237 & 1.156 & 273.3 & 12.41 & - & - & 4.83 & - & - & 207 & 0.778 \\
\hline 270 & 0.872 & 6.6 & 13.36 & 13.39 & 13.74 & 2.05 & $2.7 \pm 0.2$ & $>3.2$ & 94 & 0.927 \\
\hline 275 & 1.233 & 253.2 & 10.64 & 10.75 & 10.92 & 4.45 & $>4.6$ & $>4.6$ & 94 & 0.879 \\
\hline 297 & 0.775 & 120.4 & 10.62 & 10.69 & 11.01 & $5.2 \pm 0.1$ & $>6.0$ & $>6.0$ & 102 & 0.973 \\
\hline 362 & 0.959 & 261.3 & 13.41 & 13.60 & 14.03 & 3.69 & $4.6 \pm 0.2$ & $>4.4$ & 148 & 0.862 \\
\hline 487 & 0.611 & 323.8 & 12.45 & - & - & 3.25 & - & - & 34 & 0.987 \\
\hline
\end{tabular}

are very likely to be real systems. It is also likely that some systems with lower probabilities are real as well, even though one of the physically unrelated pairs identified above (W 339) has an uncomfortably high probability $(99.2 \%)$ of being real from a purely statistical analysis. This is a reminder that as much information as possible should be used to ascertain membership and that one should not place reliance on statistical arguments alone in individual cases. If we consider all binaries down to a $2 \sigma$ threshold while excluding those rejected as projected systems (including from Sect. 3.2), we are left with 11 candidate cluster binaries in our Main Sample. Both this number and the six "very high probability" binaries are considered in further sections.

\subsection{Masses of the detected companions}

In this section, we use current theoretical evolutionary models to estimate the mass of the detected secondaries which, in turn, allows us to derive binary mass ratios. The resulting secondary masses, derived from the $2 \mathrm{Myr}$ mass-luminosity relation constructed below, are summarized in Table 4 . In principle, the mass of every cluster member, including the primaries, can be derived from this mass-luminosity relation and our near-infrared photometry. However, the variable and anomalous extinction towards the cluster prevents this, and so we are obliged to take a more indirect route.

With regard to the primaries, those of known spectral type are easily handled by using the theoretical relation between mass and effective temperature/spectral type for massive stars. We have used models from Schaller et al. (1992) to estimate masses for stars already on the main sequence, together with spectral type-effective temperature conversion tables from Böhm-Vitense (1981). Premain sequence evolutionary models are needed for stars with masses below $4 M_{\odot}$, i.e., those of spectral type later than B5, since they are still contracting. For those 


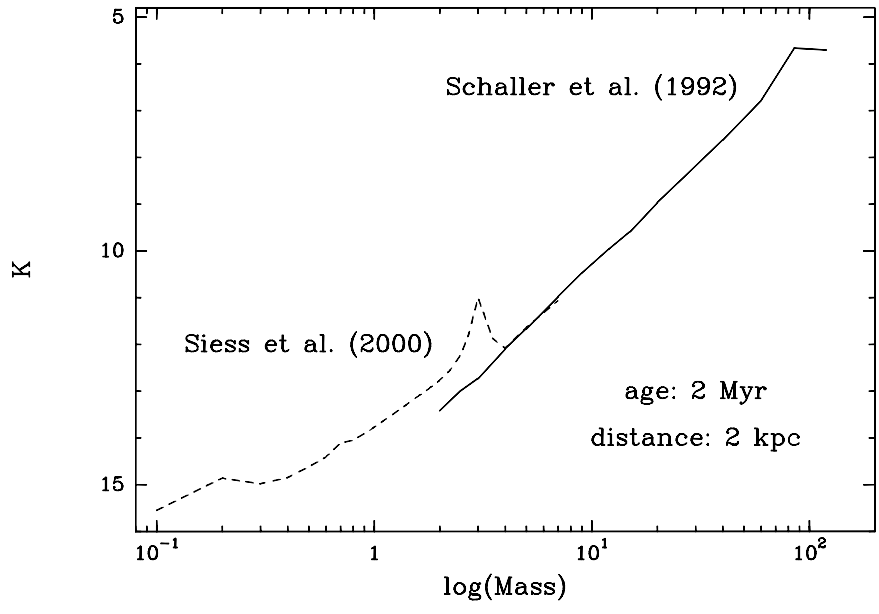

Fig. 5. Theoretical $K$-band unreddened magnitude-mass relationship for 2 Myr-old stars, as used in this work. For masses below $7 M_{\odot}$, we used the Siess et al. (2000) evolutionary models which take pre-main sequence evolution into account. For higher masses, the models of Schaller et al. (1992) were used in conjunction with bolometric corrections from Kenyon \& Hartmann (1995) and Böhm-Vitense (1981).

objects, we have used models from Siess et al. (2000), which extend from $7 M_{\odot}$ down to $0.1 M_{\odot}$. Both sets of models are in good agreement over the common 4-7 $M_{\odot}$ mass range. In the last stages before reaching the main sequence, stars undergo a rapid phase of contraction and exhibit a strong dependence of effective temperature on mass: a $3 M_{\odot}$ star has a spectral type G0, while a $3.5 M_{\odot}$ star is already at B7, according to the Siess et al. (2000) models. Consequently, we did not estimate the exact mass of the primaries with spectral types later than B5; rather a 3-4 $M_{\odot}$ range was considered. The primaries of $\mathrm{W} 224$ and W 260 have unknown spectral types, so that the mass ratio of these systems cannot be estimated.

To derive masses for the companions, we first constructed theoretical $J$-, $H$ - and $K$-band mass-luminosity relations from the Siess et al. (2000) and Schaller et al. (1992) models. The $K$-band relation is presented in Fig. 5 . Given the mass of the primary implied by its spectral type (as described in the previous paragraph), we estimated the "theoretical" brightness of the primary through the above relation, we then used the observed flux ratio to infer the "theoretical" brightness of the companion, and then we re-entered the above relation to obtain the mass of the secondary star. The underlying assumption to this method, of course, is that both stars are identically embedded, i.e., the extinction is mostly due to the molecular cloud itself.

For real companions, the masses estimated independently from different filters should (and apparently do) agree well with each other, while significant variations and/or very low masses are found in the case of likely background companions, because of their heavier extinction and extremely red colours. The slight changes in mass with wavelength listed for W 223 and W 267 in Table 4 can be explained by photometric uncertainties and/or
Table 4. Masses of both components in all binaries of our Main Sample with a primary of known spectral type. The three estimates for the secondaries were independently obtained in each filter. For some primaries (W 243, W 299, W 400) and secondaries (W 175, W 311), we only give a range of possible mass because of the ambiguity discussed in the text. The last column gives the average binary mass ratios and is left empty for the non-physical systems. Because the flux ratio of the W 299 system is so close to unity in all filters, we assumed the mass ratio to be $q=1$.

\begin{tabular}{llllll}
\hline star & $M^{\mathrm{A}}$ & $M_{K}^{\mathrm{B}}$ & $M_{H}^{\mathrm{B}}$ & $M_{J}^{\mathrm{B}}$ & $q=\frac{M_{\mathrm{B}}}{M_{\mathrm{A}}}$ \\
& {$\left[M_{\odot}\right]$} & {$\left[M_{\odot}\right]$} & {$\left[M_{\odot}\right]$} & {$\left[M_{\odot}\right]$} & \\
\hline \hline W 25 & 12.5 & 2 & - & - & 0.16 \\
W 175 & 33.5 & $2-5.5$ & $2.7-4.6$ & - & $0.08-0.14$ \\
W 188 & 12.5 & 2.4 & 2.4 & 2.6 & 0.2 \\
W 205 & 38 & 16.5 & 16.0 & - & 0.42 \\
W 223 & 10 & 0.10 & 0.13 & - & $\sim 0.01$ \\
W 227 & 8.8 & 0.7 & - & - & 0.08 \\
W 254 & 10 & 6 & 6 & 6 & 0.6 \\
W 267 & 7.7 & 1.3 & 1.3 & 1.0 & 0.15 \\
W 299 & $3-4$ & $3-4$ & $3-4$ & $3-4$ & 1.0 \\
W 311 & 6.4 & $2-5$ & $2.8-5$ & $3-5$ & $0.47-0.78$ \\
\hline \hline \multicolumn{7}{c}{ Background companions from Sect. 3.1 } \\
\hline W 243 & $3-4$ & $1.3-0.7$ & $0.9-0.45$ & $0.2-0.15$ & - \\
W 313 & 4 & $<0.1$ & $<0.1$ & $<0.1$ & - \\
W 339 & 5.8 & 0.95 & 0.66 & - & - \\
W 343 & 10 & $<0.1$ & $<0.1$ & $<0.1$ & - \\
\hline \hline \multicolumn{7}{c}{ Background companions from Sect. 3.2 } \\
\hline W 290 & 7.7 & $<0.1$ & - & - & - \\
W 400 & $3-4$ & $<0.1$ & $<0.1$ & $<0.1$ & - \\
\hline
\end{tabular}

moderate differential extinction while that observed for W 339 is significant. In two systems not already rejected, W 290 and W 400, the companions are so faint that they are brown dwarf candidates if they belong to the cluster. Alternatively, both companions may be background giant stars, which is also suggested by their low probabilities of being real companions, below the $2 \sigma$ confidence level. Both systems have been flagged as candidate background companions in Table 3. The companion to W 400 appears only slightly more extincted than its primary by $\Delta A_{V} \sim 2-3$ mag while W 290 was only detected in the $K$-band, therefore its colours could not be used to reject it as a non-physical system.

From this study, we confirm as likely members most of the companions discovered in this study and not already discarded as background objects. On the other hand, we consider the companions to W 290 and W 400 to be likely background stars, bringing the total number of suspected background stars to seven. As a by-product, we find mass ratios ranging from unity down to $q \lesssim 0.1$, with primary masses in the range $3-40 M_{\odot}$. Only two companions in our sample appear to be high-mass stars (W 205 B and W $254 \mathrm{~B}$, photometric spectral types O9 and B3 respectively), and over half of the binaries have $q \lesssim 0.2$. This suggests that low mass ratios are indeed extremely common in 
high-mass binaries. A consideration of $3 \sigma$ systems yields a median mass ratio as high as $q \sim 0.5$, because very faint companions have a higher probability of being due to random pairing due to the increasing surface density toward fainter objects. On the other hand, a significant number of low-mass companions may be unaccounted for when such a conservative criterion is applied. There is no evidence for a change in mass ratio distribution with primary mass in our sample, although small number statistics prevent any detailed analysis.

We finally note that among the five stars of our Main Sample that are supposed to have M-type companions according to de Winter et al. (1997) based on optical/nearinfrared spectral energy distribution fitting, two appear to be single in our survey (W 262 and W 273), one other we identify as a chance projection binary (W 339), and the remaining two have companions that were detected here but with intermediate- to early-type stars (W 299 and W 188). Only the one background companion seems to be red enough to be responsible for the observed nearinfrared excess, suggesting that the other two systems may be triple systems or that the significant near-infrared excess of their primaries has some other cause than the presence of a close companion.

\subsection{Overall binary frequency in NGC 6611}

Among the 60 targets of our Main Sample, 18 are found to have a visual companion within $1^{\prime \prime} .5$, the systems being evenly spread throughout the cluster. Of these, seven are rejected as projected background objects by virtue of their infrared photometric properties and/or apparent mass ratios. We are thus left with eleven $2 \sigma$ candidate binaries, which implies an observed binary frequency of $18 \pm 6 \%$ over the 200-3000 AU separation range. Restricting our sample to stars with OB spectral type or to those located in the core of the cluster does not significantly alter this estimate: the binary frequencies in those two subsamples are $20 \pm 6 \%$ and $19 \pm 6 \%$. Finally, a conservative lower limit to the binary frequency among high-mass stars in NGC 6611 can be obtained by retaining only the systems that are above the $3 \sigma$ confidence level, as explained in Sect. 3.1.2; for our Main Sample, that lower limit is $10 \pm 4 \%$.

An important limitation of our survey is closely related to the separation-dependent detection limit: we do not detect as many very close binaries as we do for wider systems. Note, however, that the apparent dearth of binaries seen in Fig. 3 at short separation could partly be the result of a geometrical effect: the area of the annulus extending from 0.1 to $0 . .5$ represents only about $10 \%$ of the total surveyed area. It would be valuable to estimate a completeness correction similar to those derived in low-mass binary surveys (Bouvier et al. 1997; Duchêne et al. 1999). However, such corrections require that we make various assumptions concerning the binary mass ratio and orbital period distributions. While they are fairly well known for solar-type binaries, no similar unbiased, well-defined distributions have been obtained for OB stars thus far. It is thus impractical for us to perform such a correction for our survey of NGC 6611.

Restricting the separation range considered here to $0.5-1$.'5 (1000-3000 AU), on the other hand, proves to be a useful step. In this range, any binary with a flux ratio $\Delta K \lesssim 3.5$ can be detected, and the average detection limit over this separation range is $\Delta K \approx 5.5$. For a typical star in our sample (median spectral type B1, $M=10 M_{\odot}$ ), such flux ratios correspond to secondary masses of about $1 M_{\odot}$ and $0.1 M_{\odot}$, respectively. We were thus able to detect any binary with mass ratio $q \geq 0.1$, as well as a large fraction of lower-mass stellar companions. Once restricted to $\mathrm{OB}$ stars to ensure sample homogeneity, we find $B F_{\mathrm{OB}}^{\mathrm{vis}}=14 \pm 5 \%$ and $7 \pm 3 \%$ when counting systems above the $2 \sigma$ and $3 \sigma$ confidence levels, respectively.

It is not possible to evaluate properly the total binary frequency of high-mass stars, due to the limited sensitivity of current surveys and because binaries with separations larger than $\sim 10$ AU but smaller than 200 AU cannot be detected either spectroscopically or by current imaging surveys. However, we can obtain a rough estimate by complementing our visual binary frequency with that of Bosch et al. (1999), i.e., assuming that the proportion of spectroscopic binaries that they found in NGC 6611 can be extended to our whole sample. They found a minimum of three binaries out of ten stars; a somewhat higher frequency is quoted by Mermilliod \& García (2001). These numbers imply a binary frequency for high-mass stars in NGC 6611 of $B F_{\mathrm{OB}}^{\text {tot }} \gtrsim 45 \%$. Even though this estimate is affected by significant uncertainties due to small number statistics, the true frequency is likely much higher, given the narrow range considered in our survey, the limited sensitivity of both surveys, and the existence of two additional binary candidates suggested by Bosch et al. (1999). It is thus very likely that most, if not all, OB stars in NGC 6611 have at least one stellar companion. Finally, W 175 is identified as a hierarchical triple system, comprised of two O stars forming a spectroscopic binary (Bosch et al. 1999) and accompanied by a distant, third, A-F component that has now been discovered in this survey.

In line with previous studies, these results point toward a larger binary frequency among OB stars than in any solar-type binary population. For instance, Duquennoy \& Mayor (1991) found a total binary frequency in field G dwarfs of about $B F_{\mathrm{G}}^{\text {tot }} \approx 60 \%$, considering 10 orders of magnitude in orbital periods and all companions down to $q=0.1$. This is only slightly higher than the estimate presented above, although the latter is restricted in its range of orbital periods, as explained above. Excluding those systems in our survey having extremely low mass ratios, $q<0.1$, which may bias the comparison, from our $2 \sigma$ systems, we find $B F_{\mathrm{OB}}^{\mathrm{vis}}(q \geq 0.1)=10 \pm 4 \%$ while the corresponding value for field $\mathrm{G}$-stars is $B F_{\mathrm{G}}^{\mathrm{vis}}(q \geq 0.1) \approx 4 \%$ in the 1000-3000 AU range. This represents a marginally significant overabundance of wide visual binaries among OB stars of a factor of about 2.5 with respect to field $G$ dwarfs. It is unlikely that the actual excess is much larger 
since our survey should be complete in these separation and mass ratio ranges, as discussed above.

The binary frequency we find in NGC 6611 can also be compared to previous imaging surveys of other high-mass star populations, although the later suffer from various selection biases and varying detection limits. Uncomplete surveys of field OB stars by Lindroos (1985), Abt et al. (1990), and Mason et al. (1998) consistently yielded binary frequencies on the order of $7-10 \%$ in the separation range 1000-3000 AU. More recently, Bouvier \& Corporon (2001) and Hubrig et al. (2001) used adaptive optics imaging to search for close companions among the Herbig AeBe and $\mathrm{X}$-ray selected late-B stars, respectively. The proportion of companions among these populations are both about $15 \%$ in the same separation range when considering only secondaries that could be found with our detection limit, a value similar to our finding in NGC 6611 although one might expect the X-ray selected sample to be biased towards higher binary rates. The only other population of clustered high-mass stars surveyed so far is the Trapezium OB stars (Preibisch et al. 1999). Because it is much closer to the Sun than NGC 6611, the separation range common to both surveys is restrained to 200-800 AU over which our NGC 6611 images have a very limited detection limit. From their sample of 13 targets, only two companions dectected by Preibisch et al. would have been detected if the Trapezium was at the same distance as NGC 6611, yielding a restricted binary frequency that is not significantly different from our findings over that separation range.

To summarize, we conclude that the frequency of wide companions among high-mass stars in NGC 6611 is similar to that observed in several other populations. A high proportion of spectroscopic binaries is observed in both NGC 6611 and the field populations (Abt et al. 1990): the $30 \%$ frequency of spectroscopic binaries in NGC 6611 revealed by Bosch et al. (1999), once again limited by the small size of their sample, is already at least comparable to that of the field G dwarfs, which host a proportion of binaries that is about $22 \%$ for periods shorter than 30 years (Duquennoy \& Mayor 1991). Overall, there is a convergent array of evidence to suggest that the binary rate is enhanced in OB stars by comparison with solartype stars in the field, independent of the environment in which these massive stars are found (whether clusters or as isolated field stars) although none of the individual studies allows a firm conclusion due to small sample sizes, inhomogeneous samples and/or selection biases.

\subsection{Other binary properties}

Correlations between binary properties and particular stellar characteristics, especially stellar mass, may be of equal or even potentially greater significance than the raw binarity rate itself. Preibisch et al. (1999) suggested that the most massive stars in the Trapezium cluster host more companions than do the stars later than spectral type
B3 or so. However, this trend vanishes when one considers only the companions that are at least $200 \mathrm{AU}$ from their primaries, i.e., at separations similar to those probed by our survey, which shows no evidence of such a trend. Indeed, we can split our sample into two roughly equal subsamples: 29 stars of spectral type B1 or earlier, and 25 stars of later spectral type. We then find no statistical difference between the binary frequencies of those subsamples $(21 \pm 8 \%$ and $16 \pm 8 \%)$.

Most of the binaries detected in our survey have small mass ratios, in good agreement with results for field star surveys (Abt et al. 1990; Mason et al. 1998). The latter studies, as well as the Preibisch et al. (1999) study of the Trapezium, concluded that the mass ratios of visual binaries can be reproduced by assuming the secondary masses are taken at random from the universal initial mass function (IMF). Given our inefficiency in detecting very low-mass ratio systems and our statistical limitations, we cannot confirm whether this conclusion applies to NGC 6611. On the other hand, the mass ratio distribution for high-mass spectroscopic binaries appears to be much flatter (e.g., Mason et al. 1998), which suggests that two different formation processes are involved; however, spectroscopic surveys are known to be relatively inefficient in detecting low-mass secondaries. In conclusion, the IMF distribution of secondary masses seems reasonable for visual binaries in all samples studied so far. We also note that, although long-period solar-type binaries show an almost flat mass ratio distribution, the latter is consistent with an IMF pairing of companions (Duquennoy \& Mayor 1991) (at least down to $q=0.2$ ), which in turn may suggest that the formation mechanism of binary systems is not dramatically different for low- and high-mass stars.

Among other properties that could be linked to binarity is the line emission in the spectra of these massive stars. Emission lines have been identified in many of the B stars of this cluster, and we identify 28 emission stars in our sample from the results of Thé et al. (1990), Hillenbrand et al. (1993) and de Winter et al. (1997) (for a contrary view, cf. Herbig \& Dahm 2001). Four of these stars were found to have companions in our survey, representing a binary frequency of $14 \pm 7 \%$ among this subsample, which is indistinguishable from the frequency derived for the sample as a whole. It thus seems that the presence of a wide companion is unrelated to the emission properties of B type stars in NGC 6611.

Finally, through a full coverage of the optical and nearinfrared domains, Hillenbrand et al. (1993) and de Winter et al. (1997) have identified stars possessing significant near-infrared excess. Such excesses can be related to the presence of a circumstellar disk, to an abnormal extinction law, or to the existence of a close red M-type companion to the stars (de Winter et al. 1997). Although the exact origin of this excess is unknown in many case, it is interesting to note that the proportion of binary systems among stars having a near-infrared excess is similar to that of the full sample (two binaries among twelve targets, or $\sim 17 \%$ ). The presence of a companion thus seems independent of 
any source of near-infrared excess, such as a circumstellar disk.

\section{Implication for the formation mechanism of high-mass stars}

The main finding of our survey is that wide binaries are rather common among massive stars in a cluster like NGC 6611. In the framework of the model presented by Bonnell et al. (1998), which seeks to account for the formation of massive stars through numerous mergers, this result raises at least two related questions: How exactly do these systems form? How do they survive for at least a couple of million years despite the many close-by interactions they have with other cluster members? By comparison, the accretion process suggested by Nakano (1989), which takes place through an equatorial disk and assumes that each massive protostar is relatively isolated, has little difficulty retaining companions once they are formed. How such configurations would form to start with nonetheless remains unclear. In this section, we first consider the issue of the survival of wide binaries in dense stellar environments, and then we try to understand how such systems fit into the framework of our current knowledge of massive star formation.

\subsection{Survival of wide massive binaries in dense clusters}

The disruptive effects of the dynamical evolution of dense clusters have been studied in detail through numerical simulations over the last years (Kroupa 1995; Kroupa et al. 1999, 2001). Although these works were primarily interested in the evolution of low-mass binaries, some general conclusions can be extended to high-mass stars. First, the timescale over which wide binaries are significantly depleted is the cluster crossing time. Second, markedly different behaviours are expected for "soft" and "hard" binaries, the division between the two types being those systems whose binding energy is equal to the average (translational) kinetic energy of the cluster constituents.

The crucial parameters here, the crossing time and the velocity dispersion, are currently unknown in NGC 6611, both because the low-mass population, hence the total mass of the cluster, has not yet been probed, and because of the very small proper motion of the cluster members. Still, we can derive a rough estimate of the soft/hard boundary if we arbitrarily assume that the velocity dispersion of NGC 6611 is similar to that of the Trapezium cluster $\left(\sigma \sim 2 \mathrm{~km} \mathrm{~s}^{-1}\right)$, which is the best known massive cluster of comparable age.

Kroupa (1995) has shown that the distinction between soft and hard systems in the Trapezium cluster occurs at separations of the order 1000 AU for solar-type primaries; a striking lack of systems wider than this limit has been subsequently documented by Scally et al. (1999). Since in our survey we consider primaries that are on average 10 times more massive, this limit is pushed outwards to about 3500 AU in NGC 6611 because of the deeper potential well. This value is somewhat larger than the widest systems we measure here. However, according to the model results, systems with separations that are a significant fraction of this limit should also be greatly depleted in a few crossing times, although not completely (Kroupa et al. 2001).

Qualitatively, we can say that binaries with separations as large as 3000 AU can survive in an environment as dense as the Trapezium cluster or NGC 6611, although some of the primordial systems may already have been destroyed. Provided wide system can form during the highmass star formation process, it is thus not surprising that they are able to live for a few million years. Even so, it is likely that there were even more wide massive binaries early on in the cluster history than exist now, and this may be harder yet to reconcile with the merger model, as we discuss below.

\subsection{High-mass star formation: Merging versus accretion processes}

The model proposed by Bonnell et al. (1998) to explain the formation of massive stars is based on dramatic dynamical events, which involve mergers of intermediate mass protostellar cores. In this framework, most of the mergers would occur before the cores have contracted into stellar objects, at an early epoch in the cluster history when the environment is extremely dense. Nevertheless, most two-body interactions do not lead to mergers but rather to close encounters. The latter can form binary systems through the influence of tidal forces, which may be strong enough to brake a grazing fragment down to a bound orbit if its periastron is only a few stellar radii, as described by Bonnell et al. (1998). Then, because these protobinaries form very early in the process, subsequent accretion onto the system will likely feed both seeds at similar rates and yield a tight binary that is comprised of two relatively massive stars. The terminal state at the end of this process qualitatively matches the observations of high-mass spectroscopic binaries described above. However, tidal interactions would not appear to be efficient enough to form binaries as wide as the visual binaries in our survey, and so an explanation for the existence of those binaries is still needed.

Consider the following picture. Let us assume that for a massive star to form as the product of tidal interactions, the stellar density during the merging process was necessarily much higher than it is now (so as to ensure a large enough number of mergers). Therefore, any wide binary formed early on in the cluster history would have been rapidly disrupted, and the systems that are still present in the cluster must have formed after the density dropped significantly, i.e., after the merging period ended. One possibility is that a fragment might be captured at the time of the last merger as a result of a dissipative three-body interaction. In that case, the mass of the wide companions 
would roughly follow that of the fragments, hence the IMF, which is not inconsistent with the observations. Of course, this is an ad hoc explanation and it may be unrealistic to assume that encounters and mergers stopped so abruptly unless gas removal from the cluster core occurs on a timescale at least as short as the merger frequency.

Another possibility is that tidal interactions during mergers and/or grazing encounters are able to disrupt the fragments before they merge, and thus lead to the formation of a significant accretion disk around the massive protostar. Subsequently this disk might disintegrate into smaller fragments and in the process form a low-mass companion. Observational evidence shows that disks as large as several thousand AU do exist (e.g., O'Dell \& Wen 1994; van der Tak et al. 2000). Due to their spatial extent and viscous properties, disks are more difficult to sweep out completely through a nearby encounter than it is to eject a similarly massive companion. Indeed, the perturbation induced by an object that is passing by could trigger the fragmentation of the disk into a secondary component. Hence, the disks could survive for a long time, even if they form in the early phases of the cluster creation. However, the frequency of such large disks is currently unknown and may be too low to account for the observed large proportion of wide companions.

Overall, the violent process suggested by Bonnell et al. (1998) to describe the formation of massive stars agrees with the observed properties of spectroscopic binaries, but presents some difficulties with regard to much wider systems. Another difficulty is the similarity in the binary properties of the B stars in the cluster and field populations. While the former would have experienced mergers, the latter are more likely to have formed via accretion in quieter and looser environments, since most of them are not identified as runaway stars from some massive cluster and therefore probably formed from a small molecular cloud. Such different formation paths would appear unlikely to yield a similar binary frequency and properties, casting some doubt upon the model described above.

To circumvent these difficulties, let us consider the possibility that high-mass objects form through the canonical accretion process (Beech \& Mitalas 1994), even in dense clusters. In this scenario, high-mass stars are surrounded by accretion disks which, again, may fragment into a stellar companion. The disks need to have a mass that is comparable to that of the central star in order to account for both the triggering of the gravitational instability and the existence of massive companions in such an accretion scenario. Subsequent accretion would again probably lead to the formation of a system consisting of two high-mass stars as the disk would likely become unstable very fast. However, the early fragmentation of the disk would prevent the central object from reaching a high mass by halting the viscous phenomenon. Similarly, binaries could form through the fission of a collapsing envelope, before radiation pressure dissipates it. Again, this would occur very early in the formation process and would lead to equal mass systems for the same reasons as above. The large number of unequal mass systems does not seem to fit very well with both of these models.

Alternatively, visual binaries could simply result from the fragmentation of the molecular cloud into two bound, widely separated prestellar cores that would subsequently collapse, as is supposed to be the case for low-mass binaries. Indeed, the objects have to be fairly isolated for the accretion process to proceed until the star reaches a mass $M>10 M_{\odot}$. Therefore, this model requires that the stellar density has not been much higher in the past than it is now, in which case one does not expect strong dynamical effects from mergers or very close encounters that could disrupt these wide systems. In this picture, cloud fragmentation naturally leads to a companion mass distribution that is similar to the IMF, as observations suggest for both high- and low-mass field stars. As a consequence of this universal scenario, one does not expect to find dramatically different binary properties between $\mathrm{O}$ and $\mathrm{B}$ stars, again an expectation that is in agreement with the observations. The overabundance of wide binaries among high-mass stars would simply be the tracer of their enhanced capability to retain low-mass companions through their deeper potential well (see Sect. 4.1). This would explain why both cluster and field massive binaries present similar properties while the binary frequency of solar-type populations appears to be dependent upon environmental conditions and particularly the stellar density (Duchêne et al. 1999; Bouvier et al. 2001).

Although the formation process through residual gas accretion seems to match more naturally the observed massive binary properties, it is not without its own difficulties. Most importantly, one might wonder whether high-mass stars that form in a rich cluster such as NGC 6611 can be considered to be isolated throughout the accretion process, which lasts for a million years or so (Beech \& Mitalas 1994; Behrend \& Maeder 2001). This may be correct if NGC 6611 has had constant properties since its birth, and if these characteristics are similar to those of the Orion Trapezium cluster in its current stage. However, studies of the dynamical evolution of stellar clusters show that gas removal in the first few millions years causes the cluster to expand significantly (e.g. Kroupa et al. 1999). If NGC 6611 has actually been an order of magnitude or more denser than it currently is, it would seem hard to disregard the merging process as an important channel for high-mass star formation and to keep considering protostars as isolated from the rest of the cluster in which they are embedded.

\section{Conclusion}

We have used the adaptive optics system at CFHT to survey 60 high-mass stars, most of them of spectral type $\mathrm{O}$ or $\mathrm{B}$, to look for companions in the projected separation range $200-3000 \mathrm{AU}\left(0^{\prime \prime} 1-1{ }^{\prime \prime} \cdot 5\right)$. This is the first visual binary survey among such a large and homogeneous sample of high-mass stars. We identified 11 likely physical systems with flux ratios as large as $\Delta K \gtrsim 5$ mag. This yields 
an uncorrected binary frequency of $18 \pm 6 \%$ in the orbital period range $10^{3} \lesssim P($ yrs $) \lesssim 5 \times 10^{4}$. Limiting our search to binaries with mass ratios $q \geq 0.1$, we find a marginally significant overabundance of companions among OB stars in NGC 6611 than in the field population of solar-type dwarfs, reinforcing the trend that high-mass stars host more wide companions than do lower-mass objects, both in the visual and spectroscopic domains.

Mass ratios have been derived for every system whose primary spectral type is known. It appears that half of the detected binaries have $q \lesssim 0.2$, pointing to a large predominance of small mass ratios in wide massive binaries. This behaviour is in rough agreement with previous surveys, which have generally favored a random association of companions from the initial mass function with massive primaries. No obvious correlation is found between binarity and primary mass, propensity toward emission lines, or infrared excess of the system.

The high rate of occurence of wide systems (several hundred AU) among massive stars seems to contradict the formation model based on mergers that has been proposed by Bonnell et al. (1998). Although this model qualitatively predicts the properties and high frequency of spectroscopic binaries, the numerous encounters between protostars in the early stages of the cluster evolution should succeed in disrupting most of the wide visual binaries, due to their lower binding energy. To reconcile this model with the observed visual binary frequency, the capture of a low-mass wide companion has to occur at the time of the last merging event, so that the number of subsequent interactions with other objects is small. Another possible interpretation is that an extended disk of material is created and fed by successive mergers and tight encounters, which would subsequently fragment to form a wide secondary. Both of these explanations are not very satisfactory, as they require that a large number of close encounters transpire within a very narrow range of physical parameters such as impact parameter, core sizes, masses, etc.

An alternative proposed model to account for the formation of massive stars, based on large disk accretion on the protostar, arouses fewer objections, as wide binaries would likely result from the fragmentation of the cloud itself. However, for the accretion process to last long enough and to result in a high-mass star, each system must be substantially isolated for at least a million years or so. Although this might well be the correct picture for field OB stars, it is likely that clusters are very much denser in their initial formation stages, when close encounters and maybe even mergers can play an important role in the evolution of the protostars within such regions. One way to reconcile dense environments and disk accretion processes would be, for future numerical simulations, to show that close encounters, although they strongly perturb circumstellar disks, do not shorten significantly their lifetime.

From an observational approach, the detection of numerous large circumstellar disks (with radii of several hundreds of AU) around OB stars is an expected outcome of future deep imaging programs if these objects do form through continous accretion processes. On the other hand, high-angular resolution observations of deeply embedded intermediate- and high-mass protostars are awaited to test the mergers model, in which high-mass stars should be deficient in very young clusters, before most of the mergers have occurred.

Acknowledgements. We thank J.-L. Beuzit for obtaining a subset of the data presented in this paper and L. Siess for valuable exchanges regarding evolutionary tracks. We also thank A. Ghez and C. McCabe for obtaining a Keck spectrum of one of the suspected background companions, as well as for critical comments about the paper. A prompt report from an anonymous referee helped to improve and clarify this manuscript. J.E. and J.B. acknowledge financial support through a PROCOPE exchange programme by APAPE and DAAD. G.D. was partially supported at UCLA by the NSF Science and Technology Center for Adaptive Optics, managed by the University of California at Santa Cruz under cooperative agreement No. AST-9876783.

\section{References}

Abt, H. A. 1988, ApJ, 331, 922

Abt, H. A., Gomez, A. E., \& Levy, S. G. 1990, ApJS, 74, 551

Beech, M., \& Mitalas, R. 1994, ApJS, 95, 517

Behrend, R., \& Maeder, A. 2001, A\&A, 373, 190

Belikov, A. N., Kharchenko, N. V., Piskunov, A. E., \& Schilbach, E. 1999, A\&AS, 134, 525

Belikov, A. N., Kharchenko, N. V., Piskunov, A. E., \& Schilbach, E. 2000, A\&A, 358, 886

Böhm-Vitense, E. 1981, ARA\&A, 19, 295

Bonnell, I. A., Bate, M. R., \& Zinnecker, H. 1998, MNRAS, 298, 93

Bonnell, I. A., \& Davies, M. B. 1998, MNRAS, 295, 691

Bosch, G. I., Morrell, N. I., \& Niemela, V. S. 1999, RMxAA, 35,85

Bouvier, J., \& Corporon, P. 2001, in The formation of binary stars, ed. Mathieu \& Zinnecker, ASP Conf. Ser., 155

Bouvier, J., \& Duchêne, G. 2001, in The Origins of Stars and Planets: The VLT View, ed. Alves, \& McCaughrean, ESO Astrophys. Symp.

Bouvier, J., Duchêne, G., Mermilliod, J.-C., \& Simon, T. 2001, A\&A, 375, 989

Bouvier, J., Rigaut, F., \& Nadeau, D. 1997, A\&A, 323, 139

de Winter, D., Kouis, C., Thé, P. S., et al. 1997, A\&AS, 121, 223

Doyon, R., Nadeau, D., Vallée, P., et al. 1998, Proc. SPIE, 3354,760

Duchêne, G., Bouvier, J., \& Simon, T. 1999, A\&A, 343, 831

Duncan, J. C. 1920, ApJ, 51, 4

Duquennoy, A., \& Mayor, M. 1991, A\&A, 248, 485

Garmany, C. D., Conti, P. S., \& Massey, P. 1980, ApJ, 242, 1063

Ghez, A. M., Neugebauer, G., \& Matthews, K. 1993, AJ, 106, 2005

Gieseking, F. 1982, A\&AS, 49, 673

Herbig, G. H., \& Dahm, S. E. 2001, PASP, 113, 195

Hillenbrand, L. A., \& Hartmann, L. W. 1998, ApJ, 492, 540

Hillenbrand, L. A., Massey, P., Strom, S. E., \& Merrill, K. M. 1993, AJ, 106, 1906

Hubrig, A., Le Mignant, D., North, P., \& Krautter, J. 2001, A\&A, 372, 152 
Kharchenko, N., \& Schilbach, E. 1995, Astron. Nachr., 316, 91 Kroupa, P. 1995, MNRAS, 277, 1522

Kroupa, P., Aarseth, S., \& Hurley, J. 2001, MNRAS, 321, 699 Kroupa, P., Petr, M. G., \& McCaughrean, M. J. 1999, New Astron., 4, 495

Leinert, C., Zinnecker, H., Weitzel, N., et al. 1993, A\&A, 278, 129

Lindroos, K. P. 1985, A\&AS, 60, 183

Mason, B. D., Gies, D. R., Hartkopf, W. I., et al. 1998, AJ, 115,821

McAlister, H. A., Mason, B. D., Hartkopf, W. I., \& Shara, M. M. 1993, AJ, 106, 1639

McBreen, B., Fazio, G. G., \& Jaffe, D. T. 1982, ApJ, 254, 126

Meaburn, J., \& White, N. J. 1982, MNRAS, 199, 121

Mermilliod, J.-C., \& García, B. 2001, in The formation of binary stars, ed. Mathieu \& Zinnecker, ASP Conf. Ser., 191

Morrell, N., \& Levato, H. 1991, ApJS, 75, 965

Nakano, T. 1989, ApJ, 345, 464

O'Dell, C. R., \& Wen, Z. 1994, ApJ, 436, 194

Petr, M. G., Coudé Du Foresto, V., Beckwith, S. V. W., Richichi, A., \& McCaughrean, M. J. 1998, ApJ, 500, 825
Preibisch, T., Balega, Y., Hofmann, K., Weigelt, G., \& Zinnecker, H. 1999, New Astron., 4, 531

Prosser, C. F., Stauffer, J. R., Hartmann, L., et al. 1994, ApJ, 421,517

Rieke, G. H., \& Lebofsky, M. J. 1985, ApJ, 288, 618

Rigaut, F., Salmon, D., Arsenault, R., et al. 1998, PASP, 110, 152

Scally, A., Clarke, C., \& McCaughrean, M. J. 1999, MNRAS, 306,253

Schaller, G., Schaerer, D., Meynet, G., \& Maeder, A. 1992, A\&AS, 96, 269

Shu, F. H., Adams, F. C., \& Lizano, S. 1987, ARA\&A, 25, 23

Siess, L., Dufour, E., \& Forestini, M. 2000, A\&A, 358

Simon, M., Ghez, A. M., Leinert, C., et al. 1995, ApJ, 443, 625

Thé, P. S., de Winter, D., Feinstein, A., \& Westerlund, B. E. 1990, A\&AS, 82, 319

van der Tak, F. F. S., van Dishoeck, E. F., Evans, N. J., \& Blake, G. A. 2000, ApJ, 537, 283

van Dessel, E., \& Sinachopoulos, D. 1993, A\&AS, 100, 517

Walker, M. F. 1961, ApJ, 133, 438

Wolfire, M. G., \& Cassinelli, J. P. 1987, ApJ, 319, 850 